\title{
Comparability of dental implant site ridge measurements using ultra-low-dose multidetector row computed tomography combined with filtered back-projection, adaptive statistical iterative reconstruction, and model-based iterative reconstruction
}

\author{
Asma'a Abdurrahman Al-Ekrish ${ }^{1}$ (i) $\cdot$ Reema Al-Shawaf ${ }^{1} \cdot$ Wafa Alfaleh $^{1} \cdot$ Romed Hörmann $^{2} \cdot$ Wolfgang Puelacher $^{3}$. \\ Gerlig Widmann ${ }^{4}$
}

Received: 20 July 2018 / Accepted: 20 September 2018 / Published online: 13 October 2018

(c) The Author(s) 2018

\begin{abstract}
Objective To assess the linear measurements of edentulous ridges recorded from multidetector row computed tomography (MDCT) images obtained by a previously untested ultra-low dose in combination with filtered back-projection (FBP), adaptive statistical iterative reconstruction (ASIR), and model-based iterative reconstruction (MBIR).

Methods Three cadavers were imaged using a reference protocol with a standard dose and FBP (volume CT dose index (CTDIvol): $29.4 \mathrm{mGy}$ ) and two ultra-low-dose protocols, LD1 and LD2 (CTDIvol: 0.53 and $0.29 \mathrm{mGy}$ ). All test examinations were reconstructed with FBP, ASIR 50, ASIR 100, and MBIR. Linear measurements from the images of the edentulous ridges recorded from the test protocols were compared with those from the reference using a one-sample $t$ test, Bland-Altman plots, and linear regression. Statistical significance was set at a $p$ value of 0.05 .

Results The one-sample $t$ test demonstrated a statistically significant difference between the measurements from the reference protocol and all test protocols. The difference was not clinically significant for the following three test protocols: LD1/ FBP, LD1/ASIR 50, and LD2/FBP. Bland-Altman plots with linear regression showed no systematic variation between the measurements obtained with the reference protocol and these three test protocols.

Conclusions The lowest-dose protocol to demonstrate comparable measurements with a standard MDCT dose was CTDIvol $0.29 \mathrm{mGy}$ with FBP. These results must be considered with caution for areas of the jaws with thin cortication. The results in areas of thin cortication should be verified by studies with larger sample sizes at such areas and comparison with true gold standard measurements.
\end{abstract}

Keywords Anatomy, cross-sectional $\cdot$ Image-guided surgery $\cdot$ Imaging, three-dimensional $\cdot$ Multidetector row computed tomography $\cdot$ Radiation dosage

\section{Introduction}

The widespread use of computed tomography (CT) in the analysis of prospective dental implant sites, although diagnostically beneficial, may contribute to the increasing collective dose of ionizing radiation to populations [1]. Therefore,

Electronic supplementary material The online version of this article (https://doi.org/10.1007/s11282-018-0350-z) contains supplementary material, which is available to authorized users.

Asma'a Abdurrahman Al-Ekrish

aalekrish@ksu.edu.sa; asma.alekrish@gmail.com

Extended author information available on the last page of the article dose optimization of multidetector row CT (MDCT) imaging in dental implantology is needed [2].

Although cone beam CT is advocated for replacement of MDCT in implant diagnostics because of the reportedly lower radiation dose and cost, MDCT is still the only option available in some settings. Radiation doses imparted by cone beam CT and MDCT are affected by numerous factors but may be comparable in some situations, especially if ultralow MDCT doses are utilized [3-5].

One method that has been advocated because of its allowance of dose reductions in MDCT is the use of iterative reconstruction techniques (IRTs) to replace the filtered back-projection (FBP) technique in reconstruction of MDCT 
images. Two examples of IRTs are adaptive statistical iterative reconstruction (ASIR) and model-based iterative reconstruction (MBIR). Compared with FBP, the use of ASIR and MBIR have been shown to produce images with reduced noise at ultra-low doses [6]; however, they may also lead to over-smoothening of the images, which may adversely affect identification of anatomical interfaces in the images [7, 8]. Use of ASIR and MBIR, as well as FBP, with ultra-low-dose MDCT at a volume CT dose index (CTDIvol) of $0.53 \mathrm{mGy}$ has been demonstrated to produce linear measurements of dental implant sites comparable with those produced with a standard dose (CTDIvol of $36.71 \mathrm{mGy}$ ); however, ASIR and MBIR did not provide an advantage over FBP [9].

Notably, most of the samples in the previous study were mandibular sites, which have thicker cortical boundaries than maxillary sites. It is therefore conceivable that identification of ridge boundaries and recording of ridge dimensions at maxillary sites may be more adversely affected at ultra-low MDCT doses and may thus benefit from reduced noise levels produced by ASIR and MBIR. Furthermore, the lowest dose limit for production of acceptable linear measurements and which reconstruction technique would support the lowest dose reductions remain unknown.

Investigation of more aggressive dose reductions using such techniques has the potential to further reduce the radiation doses imparted by MDCT imaging of dental implant sites. However, because the effect of dose on image quality is directly influenced by the reconstruction technique used, investigations of dose reductions must also take the reconstruction technique into consideration. Therefore, this study was performed to assess the comparability of linear measurements of edentulous ridges recorded from MDCT images obtained by a previously untested ultra-low dose in combination with FBP, ASIR, and MBIR compared with a reference MDCT protocol. Testing the various combinations of doses with reconstruction techniques will help to determine which reconstruction technique allows use of the lowest dose.

\section{Materials and methods}

This study was performed using three cadaveric heads. Institutional review board approval was not required because the bodies used in the study were donated by people who had given their informed consent for their use for scientific and educational purposes prior to death and the study fulfilled all requirements necessary for studies on human cadavers according to the regulations of the Division of Clinical and Functional Anatomy, Medical University of Innsbruck $[10,11]$. The cadavers had been preserved using an arterial injection of a formaldehyde-phenol/alcohol-glycerin solution and immersion in phenolic acid in water for 1-3 months [12]. Two of the cadavers were completely edentulous, and one was partially dentulous in the mandibular anterior/premolar regions.

\section{Imaging protocols}

Each of the cadavers was imaged using a 64-row CT scanner (Discovery CT750 HD; GE Healthcare, Vienna, Austria) using a reference protocol and two ultra-low-dose test protocols, LD1 and LD2. The position of each cadaver within the gantry of the MDCT device was not changed between examinations. The exposure parameters, reconstruction technique, and imparted dose of each examination are detailed in Table 1 . The effective doses of the protocols were also calculated from the dose-length product based on a $k$ factor of 0.0021 for adult head examinations [13]. Nine MDCT datasets ( 1 reference and 8 test datasets) were obtained for each cadaver, for a total of 27 datasets. All datasets were obtained with a bone convolution kernel except for the MBIR datasets, which were obtained with a standard convolution kernel because a bone kernel is not available with MBIR. The datasets were uploaded in Digital Imaging and Communication in Medicine format to an online storage site, Google Drive; they were then downloaded and imported into a three-dimensional image reformatting software program (OnDemand Software, version 1.0; Cybermed Inc., Seoul, South Korea) for reformatting of the sample sites.

\section{Image processing}

The image processing and analysis method used was similar to that described in a previous study [9]. Processing and reformatting of all sample images were performed by one maxillofacial radiologist (A.A.) with 12 years of experience in MDCT image reformatting. The entire lengths of the maxillary and mandibular edentulous areas were used to obtain the cross-sectional sample images. The exclusion criteria for the sample sites were areas containing foreign objects or artificial defects and areas with buccolingual or occlusoapical dimensions of $<2 \mathrm{~mm}$.

Each sample image was reformatted individually using the three-dimensional module of the OnDemand software. Because the position of each cadaver was fixed during the MDCT examinations, the default position and angulation of the sectional planes were identical in all datasets of each cadaver. Thus, the measured shift and angulation of the image planes allowed the production of sample sites with a standardized location and orientation between different examination protocols. The reformatting protocol for each cadaver is available upon request. The $\mathrm{mA}$ and $\mathrm{kVp}$ values were turned off, and each sample site was saved by bookmarking in the OnDemand software on the hard drive of the computer. Table 2 shows the images of one sample site obtained by the reference and test protocols. 
Table 1 List of exposure parameters and reconstruction techniques used in the reference and ultra-low-dose test protocols

\begin{tabular}{|c|c|c|c|c|c|c|c|c|c|c|}
\hline \multirow[t]{2}{*}{ Exposure protocol } & \multirow{2}{*}{$\begin{array}{l}\text { Reconstruc- } \\
\text { tion tech- } \\
\text { nique }\end{array}$} & \multirow[t]{2}{*}{ Matrix } & \multirow{2}{*}{$\begin{array}{l}\text { In-plane } \\
\text { voxel size } \\
(\mathrm{mm})\end{array}$} & \multirow[t]{2}{*}{$\mathrm{kV}$} & \multirow[t]{2}{*}{$\mathrm{mA}$} & \multirow{2}{*}{$\begin{array}{l}\text { Rotation } \\
\text { time (s) }\end{array}$} & \multirow[t]{2}{*}{ Pitch } & \multirow[t]{2}{*}{ CTDIvol (mGy) } & \multicolumn{2}{|c|}{ Effective dose $(\mu \mathrm{Sv})^{\mathrm{a}}$} \\
\hline & & & & & & & & & $\begin{array}{l}\text { Scan } \\
\text { length of } \\
5 \mathrm{~cm}\end{array}$ & $\begin{array}{l}\text { Scan } \\
\text { length of } \\
10 \mathrm{~cm}\end{array}$ \\
\hline Reference & FBP & $512 \times 512$ & 0.391 & 120 & 100 & 1.0 & 0.53 & 29.4 & 308.7 & 617.4 \\
\hline \multirow[t]{4}{*}{ LD1 } & FBP & $512 \times 512$ & 0.391 & 80 & 10 & 0.4 & 0.53 & 0.53 & 5.6 & 11.1 \\
\hline & ASIR 50 & & & & & & & & & \\
\hline & ASIR 100 & & & & & & & & & \\
\hline & MBIR & $1024 \times 1024$ & 0.195 & & & & & & & \\
\hline \multirow[t]{4}{*}{ LD2 } & FBP & $512 \times 512$ & 0.391 & & & & 0.97 & 0.29 & 3.0 & 6.1 \\
\hline & ASIR 50 & & & & & & & & & \\
\hline & ASIR 100 & & & & & & & & & \\
\hline & MBIR & $1024 \times 1024$ & 0.195 & & & & & & & \\
\hline
\end{tabular}

CTDIvol volume CT dose index, $L D$ low-dose protocol, FBP filtered back-projection, ASIR adaptive statistical iterative reconstruction, $M B I R$ model-based iterative reconstruction

${ }^{\text {a }}$ Calculated from the dose-length product based upon a $k$ factor of 0.0021

Table 2 Images of a sample site obtained with the various combinations of exposure protocols and reconstructions techniques

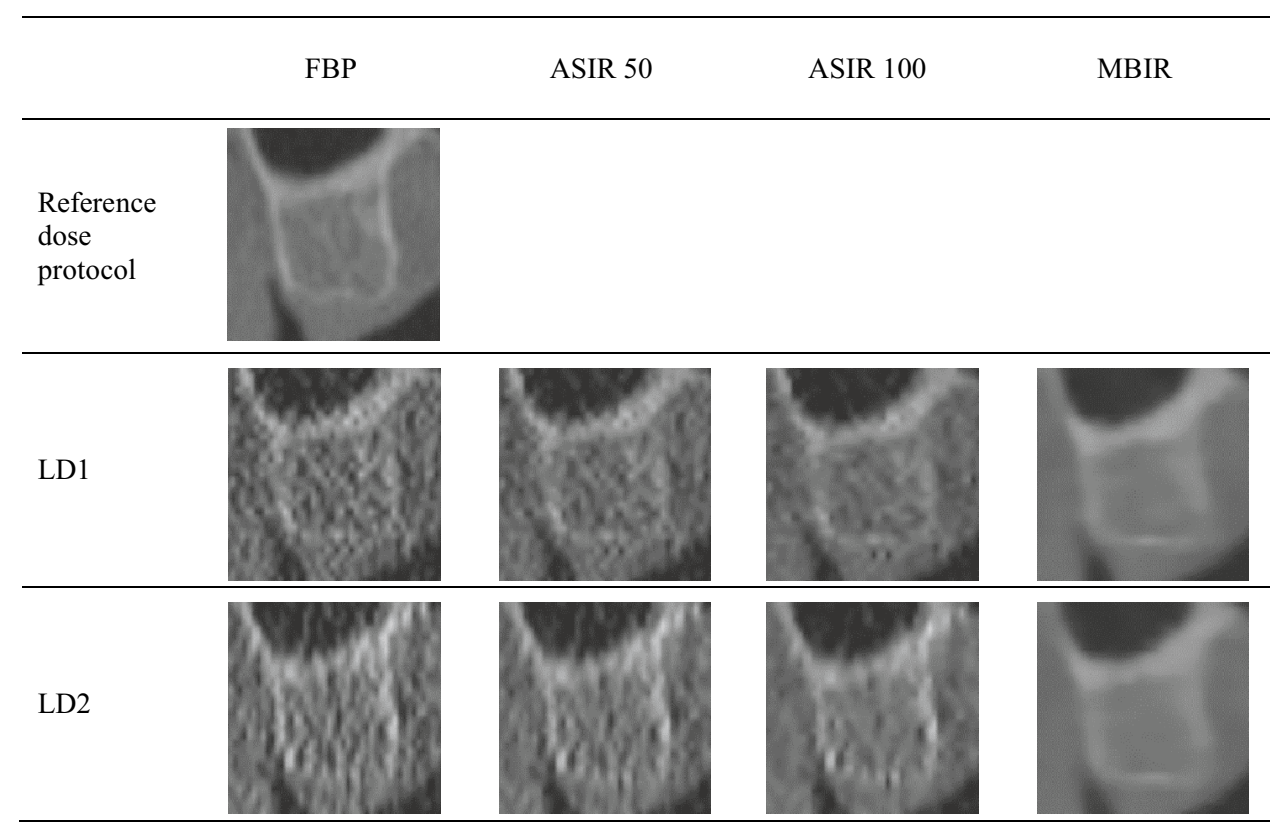

$L D$ low-dose protocol, FBP filtered back-projection, ASIR adaptive statistical iterative reconstruction, $M B I R$ model-based iterative reconstruction

\section{Image analysis}

Two oral and maxillofacial radiologists (R.S. and W.A.) with 8 and 14 years of experience, respectively, in interpretation of CT images recorded the test measurements. The examiners were blinded to the exposure protocols and reconstruction techniques and were calibrated in identification of the outer boundaries of the edentulous bone on cross-sectional images. The examiners viewed the images at a set window width/window level of 3000/650, but they were permitted to adjust the magnification level for clarity.

The height and width measurements of the ridge were recorded on the sample images along the lines representing the parasagittal and axial planes, respectively. The limits of the measurements were the outer cortical boundaries of the ridge. The apical cortical boundary at the anterior maxillary sites was the floor of the nasal cavity, and that at the posterior maxillary sites was the floor of the maxillary sinus (Fig. 1). The first examiner (R.S.) recorded all measurements 


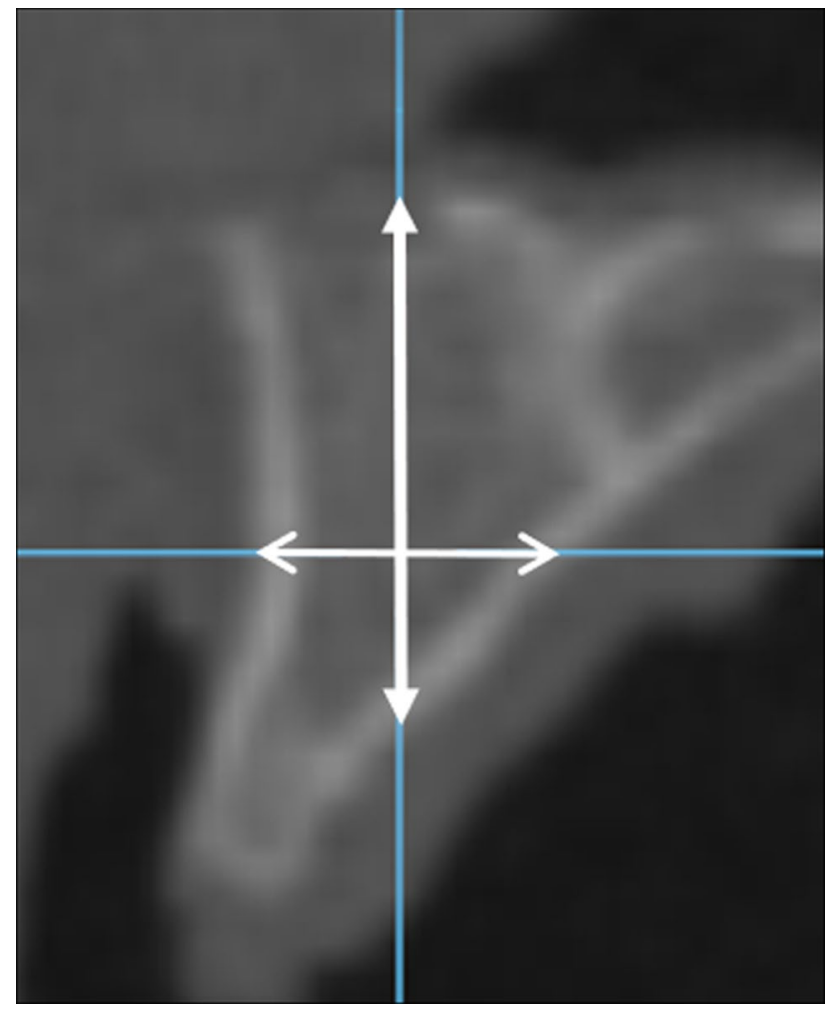

Fig. 1 Sample cross-sectional image from the anterior maxilla (acquired with the reference protocol) demonstrating the location and direction of the height and width measurements. The height measurement was recorded along the line representing the parasagittal plane (marked by closed arrows) extending from the floor of the nasal cavity to the outer surface of the cortical plate. The width measurement was recorded along the line representing the axial plane (marked by open arrows) extending between the outer surfaces of the facial and lingual cortical plates

from all of the samples once. Next, using a random number generator (http://stattrek.com/statistics/random-numbe r-generator.aspx), 50 measurements were selected for reliability testing. These measurements were then repeated by the two examiners independently of each other.

\section{Statistical evaluation}

The two measurements recorded by the first examiner were used to calculate intra-examiner reliability. For calculation of the inter-examiner reliability, the mean of the two measurements recorded by the first examiner was compared with the corresponding measurements recorded by the second examiner. Intra-examiner and inter-examiner reliability were analyzed by Cronbach's alpha and inter-item correlation.

Agreement between the measurements recorded from the reference protocol and each of the test protocols was tested by subtracting the test measurements from the reference measurements and analyzing the difference with a onesample $t$ test (test value: zero). For each test protocol, if the one-sample $t$ test revealed the absolute mean difference to be $<0.3 \mathrm{~mm}$ with a $95 \%$ confidence interval within $\pm 0.5 \mathrm{~mm}$, then it was considered that no clinically significant difference was present; in such cases, a Bland-Altman plot was constructed and linear regression was used to test for proportional bias [14]. Statistical analysis was performed using the Statistical Package for the Social Sciences (SPSS) version 24 (IBM Corp., Armonk, NY, USA). Statistical significance was set at a $p$ value of 0.05 .

\section{Results}

Thirty sample images were obtained from the three cadavers. The site distribution of the samples is shown in Table 3 . Height and width measurements were recorded from each sample for a total of 60 measurements from each imaging protocol. Nine imaging protocols were used for each sample for a total of 540 measurements.

The intra-examiner and inter-examiner reliability of the measurements were found to be very high, with Cronbach's alpha and intraclass correlation coefficients of 0.997 for the intra-examiner reliability and 0.998 for the inter-examiner reliability $(p<0.001)$.

The one-sample $t$ test revealed a statistically significant difference in measurements between the reference protocol and all the test protocols (Table 4). However, the difference was not clinically significant for the following three test protocols: LD1/FBP, LD1/ASIR 50, and LD2/FBP. Bland-Altman plots (Electronic Supplementary Fig. 1) with linear regression (Table 4) showed no systematic variation between the measurements obtained with the reference protocol and these three test protocols.
Table 3 Distribution of the sample sites

\begin{tabular}{|c|c|c|c|c|c|c|c|}
\hline \multirow[t]{2}{*}{ Cadaver } & \multicolumn{3}{|c|}{ Maxillary sites } & \multicolumn{3}{|c|}{ Mandibular sites } & \multirow[t]{2}{*}{ Total } \\
\hline & Incisor & $\begin{array}{l}\text { Canine/pre- } \\
\text { molar }\end{array}$ & Molar & Incisor & $\begin{array}{l}\text { Canine/pre- } \\
\text { molar }\end{array}$ & Molar & \\
\hline 1 & 1 & 2 & 2 & 2 & - & 4 & 11 \\
\hline 2 & 1 & 2 & 2 & 1 & - & 4 & 10 \\
\hline 3 & 1 & 2 & 2 & & 1 & 3 & 9 \\
\hline Total & 3 & 6 & 6 & 3 & 1 & 11 & 30 \\
\hline
\end{tabular}


Table 4 Differences between linear measurements obtained by the reference protocol (reference dose/filtered backprojection) and the various test protocols

\begin{tabular}{llllll}
\hline & $\begin{array}{l}\text { Mean differ- } \\
\text { ence }(\mathrm{mm})^{*}\end{array}$ & $\begin{array}{l}\text { Standard deviation of } \\
\text { difference }(\mathrm{mm})\end{array}$ & $\begin{array}{l}\text { 95\% confidence interval limits } \\
(\mathrm{mm})\end{array}$ & $\begin{array}{l}\text { Significance } \\
\text { (linear regres- } \\
\text { sion) }\end{array}$ \\
\cline { 4 - 5 } & & & Lower limit & Upper limit & \\
\hline LD1/FBP & -0.2083 & 0.61128 & -0.3662 & -0.0504 & 0.605 \\
LD1/ASIR 50 & -0.2817 & 0.66498 & -0.4534 & -0.1099 & 0.394 \\
LD1/ASIR 100 & -0.3467 & 0.61959 & -0.5067 & -0.1866 & - \\
LD1/MBIR & -0.4667 & 1.01508 & -0.7289 & -0.2044 & - \\
LD2/FBP & -0.2500 & 0.76878 & -0.4486 & -0.0514 & 0.584 \\
LD2/ASIR 50 & -0.6367 & 1.47269 & -1.0171 & -0.2562 & - \\
LD2/ASIR 100 & -0.4050 & 0.55736 & -0.5490 & -0.2610 & - \\
LD2/MBIR & -0.4583 & 1.05899 & -0.7319 & -0.1848 & - \\
\hline
\end{tabular}

- Bland-Altman plot and linear regression were not performed because the one-sample $t$ test demonstrated a mean difference greater than $\pm 0.3 \mathrm{~mm}$ with $95 \%$ confidence limits greater than $\pm 0.5 \mathrm{~mm}$

$L D$ low-dose protocol, FBP filtered back-projection, ASIR adaptive statistical iterative reconstruction, $M B I R$ model-based iterative reconstruction

*All mean differences were statistically significant $(p<0.05)$

\section{Discussion}

The present study was performed to test the accuracy of linear measurements of edentulous ridges recorded from previously untested ultra-low-dose MDCT images compared with those from a reference dose protocol. The effective doses achieved in the present study are lower than those reported for many cone beam CT devices, even for some small-fieldof-view examinations [4]. The results indicate that the measurements recorded using the lowest dose tested, CTDIvol of 0.29 , and FBP were comparable with those from a reference dose protocol. The IRTs of ASIR and MBIR did not allow similarly accurate measurements. As such, the present study demonstrates previously unrealized potentials of ultra-low reduction in doses and FBP in implant site imaging.

The images of some sample sites, especially in the maxillary posterior regions, had very thin cortical boundaries and thus subjectively appeared to be more adversely affected by the amount of noise seen with the LD2/FBP protocol. However, the boundaries of the ridge were still identifiable in the images acquired by LD2/FBP as evidenced by the objective measurements presented in Table 4 . This finding, namely that clinically useful information may be obtained with images that are not subjectively pleasing to the eye, was addressed by the National Council on Radiation Protection and Measurements when they modified the ALARA concept of imaging (as low as reasonably achievable) to the ALADA concept (as low as diagnostically achievable) [2]. The modification is meant to emphasize that regardless of whether the images are subjectively pleasing to the eye, the lowest doses should be used provided that the diagnostic information is obtainable. The European Union Council Directive 2013/59/ EURATOM also addressed this issue by mandating that for all imaging procedures, the dose of ionizing radiation should be kept as low as reasonably achievable, consistent with obtaining the required medical information [15].

The improved performance of FBP over ASIR and MBIR in the present study occurred despite smaller voxel sizes being used with MBIR. The poorer performance of ASIR and MBIR compared with FBP may be due to their oversmoothening effect, which may increase the area of blurriness at the margins of the bone [7, 8]. For the same reason, previous studies have shown that ASIR and MBIR did not improve spatial resolution or the detection of fractures $[8$, $16]$ or identification of the roof of the inferior alveolar canal [17].

In the present study, the clinical significance of the absolute measurement differences was designated as $>0.3 \mathrm{~mm}$ with a $95 \%$ confidence interval beyond $\pm 0.5 \mathrm{~mm}$ for several reasons. First, this range of error is smaller than the size of the image voxel. Second, the measurement error of the reference protocol images when compared with the gold standard of measurements recorded directly from the bone is expected to be larger than this value; previous studies that compared ridge dimensions from MDCT images acquired with the standard protocol versus the dimensions recorded directly from bone showed that the smallest mean error was $0.36 \mathrm{~mm}$ with a standard deviation of $\pm 0.24 \mathrm{~mm}$ [18-21] . Third, implant diameters may include $0.3-\mathrm{mm}$ increments, and during interpretation of images, operators commonly round off linear measurements to the nearest $0.5 \mathrm{~mm}$.

The test protocols in the present study utilized the lowest $\mathrm{mA}$ and $\mathrm{kV}$ settings possible with the device used. Thus, to further reduce the radiation dose, the rotation time was reduced to less than half of that used for the reference protocol. The combined effect of reduced $\mathrm{mA}$ and rotation time is a reduction in the amount of X-ray photons, but an increase in the quantum noise in the resultant images. However, a 
previous study demonstrated accurate ridge dimensions using the same $\mathrm{mA}$ and $\mathrm{kV}$ and rotation time settings as LD1 [9]. Therefore, to further lower the dose, the present study used a higher pitch value in LD2 than that used in the previous study. The pitch value used in the present study did not exceed the maximum acceptable pitch values for 64-row MDCT scanners, namely 0.9844 , beyond which interpolation of data occurs [22]. Although some researchers reported that the use of a pitch factor of 2 did not reduce the subjective quality of MDCT images of implant sites when combined with standard exposure parameters [23], other investigators reported that an increasing pitch factor was negatively associated with the number of successful objective linear measurements [24]. However, considering the high degree of comparability between the reference and lowest-dose protocol in the present study, investigation of further dose optimization using further increases in pitch is recommended.

The results of the present study are in agreement with a previous study that revealed comparable measurements between a reference dose protocol and an ultra-low-dose protocol similar to LD1 of the present study [9]. However, the previous study indicated that ASIR and MBIR produced measurements comparable to FBP, whereas the present study showed that FBP was more accurate at such very low doses. The reason for the differences may be that in the previous study, the sample sites were mostly in the mandibular molar region, which has a thick layer of dense cortical bone that is more clearly depicted in images and does not require image contrast as high as that needed for thin, low-density bone, which was found in other parts of the jaws in the present study. Thus, although ASIR and MBIR are beneficial in terms of noise reduction, FBP images should be used for bone measurements.

A limitation of the present study is that a site-specific analysis of the measurement differences was not conducted because of the small sample size in each specific area of the jaws. Although the overall study sample demonstrated no clinically significant difference with three of the test protocols, larger clinically significant differences might occur at particular sites with thin, low-density margins. As such, further studies with larger sample sizes at each area of the jaws and separate analysis of the measurements from each area are recommended. Additionally, the present study did not evaluate the influence of various sharpness levels of convolution kernels using ultra-low-dose images and IRTs. The use of various sharpness levels might produce different results than those obtained in the present study.

Another limitation of the present study is that the test measurements were not compared with a true gold standard of direct bone measurements. Using the reference protocol measurements as the standard must be considered with caution because such measurements have been shown to have statistically significant error [21]. Whether the measurement differences between the test and reference protocols in the present study would translate to increased or decreased error compared with bone measurements remains unknown. Therefore, further studies are recommended to investigate the absolute error of measurements compared with a true gold standard.

The lowest-dose protocol to demonstrate comparable measurements with a standard MDCT dose was found to be a CTDIvol of $0.29 \mathrm{mGy}$ with FBP. The IRTs of ASIR and MBIR yielded larger measurement differences. These results must be considered with caution when evaluating areas of the jaws with thin cortication. The results in such areas should be verified by studies with larger sample sizes in such areas, and the findings should be compared with true gold standard measurements.

Acknowledgements The authors extend their appreciation to the Deanship of Scientific Research at King Saud University for funding this work through research group number RGP-1438-037. The authors also thank all individuals who have donated their bodies and tissues for the advancement of education and research.

\section{Compliance with ethical standards}

Conflict of interest Asma'a Abdurrahman Al-Ekrish, Reema Al-Shawaf, Wafa Alfaleh, Romed Hörmann, Wolfgang Puelacher, and Gerlig Widmann declare that they have no conflict of interest.

Human rights statement This article does not contain any studies with human or animal subjects performed by any of the authors.

Open Access This article is distributed under the terms of the Creative Commons Attribution 4.0 International License (http://creativeco mmons.org/licenses/by/4.0/), which permits unrestricted use, distribution, and reproduction in any medium, provided you give appropriate credit to the original author(s) and the source, provide a link to the Creative Commons license, and indicate if changes were made.

\section{References}

1. United Nations Scientific Committee on the Effects of Atomic Radiation. Sources and effects of ionizing radiation. Official Records of the General Assembly, Sixty-third Session, Supplement No. 46; New York: United Nations; 2010.

2. National Council on Radiation Protection and Measurements. Achievements of the past 50 years and addressing the needs of the future. In: NCRP fiftieth annual meeting program; 2014.

3. Ludlow JB, Ivanovic M. Comparative dosimetry of dental CBCT devices and 64-slice CT for oral and maxillofacial radiology. Oral Surg Oral Med Oral Pathol Oral Radiol Endod. 2008;106(1):106-14.

4. Ludlow J, Timothy R, Walker C, Hunter R, Benavides E, Samuelson D, et al. Effective dose of dental CBCT - a meta analysis of published data and additional data for nine CBCT units. Dentomaxillofac Radiol. 2015;44(1):20140197. 
5. Al-Ekrish A, Alfadda S, Tamimi D, Alfaleh W, Hörmann R, Puelacher W, et al. Do ultra-low multidetector computed tomography doses and iterative reconstruction techniques affect subjective classification of bone type at dental implant sites? Int $\mathbf{J}$ Prosthodont. 2018;31(5):465-70.

6. Widmann G, Al-Shawaf R, Schullian P, Al-Sadhan R, Hormann R, Al-Ekrish AA. Effect of ultra-low doses, ASIR and MBIR on density and noise levels of MDCT images of dental implant sites. Eur Radiol. 2017;27(5):2225-34.

7. Silva AC, Lawder HJ, Hara A, Kujak J, Pavlicek W. Innovations in CT dose reduction strategy: application of the adaptive statistical iterative reconstruction algorithm. AJR Am J Roentgenol. 2010;194(1):191-9.

8. Widmann G, Dalla Torre D, Hoermann R, Schullian P, Gassner EM, Bale R, et al. Ultralow-dose computed tomography imaging for surgery of midfacial and orbital fractures using ASIR and MBIR. Int J Oral Maxillofac Surg. 2015;44(4):441-6.

9. Al-Ekrish A, Al-Shawaf R, Schullian P, Al-Sadhan R, Hörmann R, Widmann G. Validity of linear measurements of the jaws using ultralow-dose MDCT and the iterative techniques of ASIR and MBIR. Int J Comput Assist Radiol Surg. 2016;11(10):1791-801.

10. McHanwell S, Brenner E, Chirculescu ARM, Drukker J, van Mameren H, Mazzotti G, et al. The legal and ethical framework governing body donation in Europe-a review of current practice and recommendations for good practice. Eur $\mathbf{J}$ Anat. 2008;12(1):1-24.

11. Riederer BM, Bolt S, Brenner E, Bueno-Lopez JL, Cirulescu ARM, Davies DC, et al. The legal and ethical framework governing body donation in Europe-1st update on current practice. Eur J Anat. 2012;16(1):1-21.

12. Platzer W, Putz R, Poisel S. Ein neues Konservierungs- und Aufbewahrungssystem für anatomisches Material. Acta Anat (Basel). 1978;102(1):60-7.

13. Christner JA, Kofler JM, McCollough CH. Estimating effective dose for CT using dose-length product compared with using organ doses: consequences of adopting International Commission on Radiological Protection Publication 103 or dual-energy scanning. AJR Am J Roentgenol. 2010;194(4):881-9.

14. Bland JM, Altman D. Statistical methods for assessing agreement between two methods of clinical measurement. Lancet. 1986;327(8476):307-10.
15. European Union. Council Directive 2013/59/Euratom of 5 December 2013. Laying down basic safety standards for protection against the dangers arising from exposure to ionising radiation, and repealing Directives 89/618/Euratom, 90/641/Euratom, 96/29/ Euratom, 97/43/Euratom and 2003/122/Euratom. Off J Eur Union. 2014;17:13/1-13/73.

16. Widmann G, Bischel A, Stratis A, Bosmans H, Jacobs R, Gassner EM, et al. Spatial and contrast resolution of ultralow dose dentomaxillofacial CT imaging using iterative reconstruction technology. Dentomaxillofac Radiol. 2017;46(4):20160452.

17. Al-Ekrish A, Alfaleh W, Hörmann R, Alabdulwahid A, Puelacher W, Widmann G. Localization of the inferior alveolar canal using ultralow dose CT with iterative reconstruction techniques. Dentomaxillofac Radiol. 2018;47:20170477.

18. Cavalcanti MG, Haller JW, Vannier MW. Three-dimensional computed tomography landmark measurement in craniofacial surgical planning: experimental validation in vitro. J Oral Maxillofac Surg. 1999;57(6):690-4.

19. Yang J, Cavalcanti MG, Ruprecht A, Vannier MW. 2-D and 3-D reconstructions of spiral computed tomography in localization of the inferior alveolar canal for dental implants. Oral Surg Oral Med Oral Pathol Oral Radiol Endod. 1999;87(3):369-74.

20. Kobayashi K, Shimoda S, Nakagawa Y, Yamamoto A. Accuracy in measurement of distance using limited cone-beam computerized tomography. Int J Oral Maxillofac Implants. 2004;19(2):228-31.

21. Al-Ekrish A, Ekram M. A comparative study of the accuracy and reliability of multidetector computed tomography and cone beam computed tomography in the assessment of dental implant site dimensions. Dentomaxillofac Radiol. 2011;40(2):67-75.

22. Goldman LW. Principles of CT: multislice CT. J Nucl Med Technol. 2008;36(2):57-68.

23. Rustemeyer P, Streubühr U, Suttmoeller J. Low-dose dental computed tomography: significant dose reduction without loss of image quality. Acta Radiol. 2004;45(8):847-53.

24. Suomalainen A, Vehmas T, Kortesniemi M, Robinson S, Peltola J. Accuracy of linear measurements using dental cone beam and conventional multislice computed tomography. Dentomaxillofac Radiol. 2008;37(1):10-7.

\section{Affiliations}

\section{Asma'a Abdurrahman Al-Ekrish ${ }^{1}$ (D) Reema Al-Shawaf ${ }^{1} \cdot$ Wafa Alfaleh $^{1} \cdot$ Romed Hörmann $^{2} \cdot$ Wolfgang Puelacher $^{3}$. Gerlig Widmann ${ }^{4}$}

1 Division of Oral and Maxillofacial Radiology, Department of Oral Medicine and Diagnostic Sciences, College of Dentistry, King Saud University, PO Box 60169, Riyadh 11545, Saudi Arabia

2 Division of Clinical and Functional Anatomy, Medical University of Innsbruck, Müllerstrasse 59, 6020 Innsbruck, Austria
3 Department of Craniomaxillofacial Surgery, Medical University of Innsbruck, Anichstraße 35, 6020 Innsbruck, Austria

4 Department of Radiology, Medical University of Innsbruck, Anichstraße 35, 6020 Innsbruck, Austria 